\title{
Interpreting the simultaneous variability of near-IR continuum and line emission in young stellar objects
}

\author{
D.Lorenzetti $^{\text {a,* }}{ }^{\text {, S.Antoniucci }}{ }^{\text {a }}$, T.Giannini ${ }^{\text {a }}$, A.Di Paola ${ }^{\text {a }}$, \\ A.A.Arkharov ${ }^{\text {b }}$, V.M.Larionov ${ }^{\mathrm{b}, \mathrm{c}}$ \\ a INAF - Osservatorio Astronomico di Roma - Via Frascati, 33 - 00040 Monte \\ Porzio Catone, Italy \\ ${ }^{\mathrm{b}}$ Central Astronomical Observatory of Pulkovo, Pulkovskoe shosse 65, 196140 \\ St.Petersburg, Russia \\ ${ }^{\mathrm{c}}$ Astronomical Institute of St.Petersburg University, Russia
}

\begin{abstract}
We present new near-infrared (IR) spectra $(0.80-1.35 \mu \mathrm{m})$ of the pre-Main Sequence source PV Cep taken during a monitoring program of eruptive variables we are conducting since some years. Simultaneous photometric and spectroscopic observations are systematically carried out during outburst and quiescence periods. By correlating extinction-free parameters, such as HI recombination lines and underlying continuum, it is possible to infer on the mechanism(s) responsible for their origin. Accretion and mass loss processes have a dominant role in determining the PV Cep irregular variability of both continuum and line emission. The potentialities of the observational modality are also discussed.
\end{abstract}

Key words: Stars: pre-main sequence, variable, emission lines, individual: PV Cep, ISM: jets and outflows, infrared: stars

* Corresponding author.

Email addresses: dario.lorenzetti@oa-roma.inaf.it (D.Lorenzetti), simone.antoniucci@oa-roma.inaf.it (S.Antoniucci), teresa.giannini@oa-roma.inaf .it (T.Giannini), andrea.dipaola@oa-roma.inaf .it (A.Di Paola), arkadi@arharov.ru (A.A.Arkharov), vlar2@yandex.ru (V.M.Larionov). 


\section{Introduction}

Young stellar objects (YSO's) present a certain degree of irregular variability which is typically attributed to intermittent accretion (or mass loss) phenomena. According to a widely accepted picture (Hartmann \& Kenyon, 1985; Shu et al. 1994), accretion matter viscously migrates through the circumstellar disk toward its inner edge from where this matter is intermittently channeled along the magnetic field lines to the central star. The fall onto the stellar surface produces a shock that cools by emitting a hot continuum; moreover, as a consequence of the accretion event, strong winds (in some cases also collimated jets) emerge from the rotating star/disk system. Accretion-related variability may be due also to dust evaporation and condensation, a phenomenon potentially relevant in V1647 Ori (Reipurth \& Aspin 2004, Acosta-Pulido et al. 2007, Aspin 2011); V2492 Cyg (Hillenbrand et al. 2012); and PV Cep itself (Kun et al. 2011). Irregular variability is alternatively attributed to a variable extinction caused by dust clumps that occasionally intersect our line of sight (Skrutskie et al. 1996). Both mechanisms are plausible because all YSO's are expected to accrete, although with decreasing rates while the evolution goes on, and they are preferentially located in the densest parts of molecular clouds where gas and/or dust clumps are usual ingredients.

The classical observational method for studying the modalities and the causes of the irregular variability consists of monitoring the continuum emission in different spectral bands to obtain the light-curves and the colors of the fluctuations. A further (and even better) technique is to exploit simultaneously a photometric and spectroscopic monitoring in the same spectral band: this represents a powerful tool of investigation since provides extinction-free parameters that allow us to understand (i) the mechanism(s) responsible for the continuum and line emission; (ii) whether or not these mechanisms are (inter-)related; and (iii) in affirmative case, to infer on the possible common mechanism.

Remarkably, since few years we are conducting a photometric (Lorenzetti et al. 2006, 2007) and spectroscopic (Lorenzetti et al. 2009) monitoring in the near-IR (0.8-2.5 $\mu \mathrm{m}$, ZJHK bands) of a sample of low-mass pre-main sequence sources named EXor's (Herbig 1989; Reipurth \& Aspin 2010). These are targets very suitable for the aim described above, since they present large (about 3-4 mag) outbursts, characterized by a short (weeks, months) rising and a longer (months, years) declining, superposed to long (years) quiescence periods. In particular, the source PV Cep was extensively monitored (Lorenzetti et al. 2011; see also for a detailed description of this pre-Main Sequence object); its variability, firstly interpreted as due to extinction, has been later reconsidered as due to accretion and our aim here is to demonstrate (in the specific case of PV Cep) the effectiveness of the proposed approach. The novelty of 
the present short note relies on both presenting new photometric and spectroscopic data and, most importantly, considering new diagnostic parameters. Obviously, the approach adopted here for a single object (PV Cep) and for a relatively short monitoring period (about 3 years) can be ported in a more general context, once provided a meaningful monitoring of other sources.

This paper is organized in the following way: Sect. 2 shortly refers to the used instrumentation; the relevant parameters derived from previous and new observations are summarized in Sect.3; our considerations are presented in Sect.4, while final remarks are given in Sect.5.

\section{$2 \quad$ Near-IR photometry and spectroscopy}

The following analysis is based on near-IR photometric and spectroscopic data. These latter were obtained at the $1.1 \mathrm{~m}$ AZT-24 telescope located at Campo Imperatore (L'Aquila - Italy) equipped with the imager/spectrometer SWIRCAM (D'Alessio et al. 2000), which is based on a $256 \times 256 \mathrm{HgCdTe}$ PICNIC array. Photometry is performed with broad band filters $\mathrm{J}(1.25 \mu \mathrm{m})$, $\mathrm{H}(1.65 \mu \mathrm{m})$, and $\mathrm{K}(2.20 \mu \mathrm{m})$. The total field of view is $4.4 \times 4.4 \mathrm{arcmin}^{2}$, which corresponds to a plate scale of 1.04 arcsec/pixel. All the observations were obtained by dithering the telescope around the pointed position. The raw imaging data were reduced by using standard procedures for bad pixel removal, flat fielding, and sky subtraction.

Low resolution $(\mathcal{R} \sim 250)$ spectroscopy is obtained by means of two IR grisms $\mathrm{G}_{\text {blue }}$ and $\mathrm{G}_{\text {red }}$ covering the ZJ $(0.83-1.34 \mu \mathrm{m})$ and $\mathrm{HK}(1.44-2.35 \mu \mathrm{m})$ bands, respectively, in two subsequent exposures. The long slit is not orientable in position angle, and it samples a pre-defined portion of the focal plane, $2 \times 260$ $\operatorname{arcsec}^{2}$ in the east-west direction.

Long-slit spectroscopy was carried out in the standard ABBA' mode with a total integration time ranging between 800 and 1200 sec. The spectral images were flat-fielded, sky-subtracted, and corrected for the optical distortion in both the spatial and spectral directions. Telluric features were removed by dividing the extracted spectra by that of a normalized telluric standard star, once corrected for its intrinsic spectral features. Wavelength calibration was derived from the $\mathrm{OH}$ lines present in the raw spectral images, while flux calibration was obtained from our photometric data carried out on the same night of the spectroscopy.

During the monitoring period presented here, the near-IR spectra of PV Cep were taken in several occasions marked in Figure 1 with red vertical dotted 
lines. Since this object is a target included in a photometric and spectroscopic monitoring program we are performing since few years, some of these spectra have been already discussed elsewhere (Lorenzetti et al. 2009). The oservational novelty is represented by the 3 new spectra (and relative photometry) obtained in April 2010 (MJD 55310), July 2010 (MJD 55397), and August 2010 (MJD 55416) (see Figure 1). They will be commented, along with previous data, in the following Sect.4.

\section{Results}

The ZJ portion of the newly obtained (during 2010) near-IR spectra of PV Cep is plotted in Figure 2 along with our previous results. New data substantially confirm the existence of some continuum variability. HI recombination lines of the Paschen series are present: among them the $\mathrm{Pa} \beta$ appears as the most prominent in all spectra and on that line we will focus our attention. In Table 1, the $\mathrm{Pa} \beta$ observed flux, its equivalent width (EW) and the value of the underling $\mathrm{J}$ band continuum (in mag) are given for each date. Given the spectral closeness between the J-band effective wavelength $(1.25 \mu \mathrm{m})$ and the $\mathrm{Pa} \beta$ transition $(1.28 \mu \mathrm{m})$, the EW of this latter can be considered unaffected by the extinction. As such, EW is a suitable parameter to give indication on whether or not, and to what extent, the mechanisms responsible for the continuum and line emission are related (see next Sect.). The same is obviously true also for other pairs of HI recombination lines/underlying continuum: indeed we did the same analysis also for $\mathrm{Br} \gamma / \mathrm{K}$ band combination obtaining the same results not reported here to avoid any unuseful redundance.

\section{Analysis and discussion}

In the upper panel of Figure 3 an evident correlation (regression coefficient $=$ 0.93) is recognizable between the $\mathrm{Pa} \beta$ line flux and the continuum in the $\mathrm{J}$ band. Such a correlation suggests, as expected, that emission lines are generated by some mechanism strongly related to the central source. According to the lower panel of the same figure an anti-correlation seems to exist between EW and J continuum, in the sense that decreasing EW values correspond to an increasing continuum brightness. Two data points, those corresponding to J about 12 mag and marked with open symbols, seem not to follow the anticorrelation trend, but this apparent contraddiction will be discussed later. Neglecting these two points, a regression coefficient of 0.91 is obtained. Now we remark the anti-correlation indicates that, although line emission and $\mathrm{J}$ continuum are related, the latter presents an increase larger (i.e. faster) than 
Table 1

Spectroscopic and photometric parameters of PV Cep.

\begin{tabular}{ccccc}
\hline Date & MJD $^{a}$ & Pa $\beta$ Flux & EW & J band \\
& & $\left(10^{-13} \mathrm{ergs}^{-1} \mathrm{~cm}^{-2}\right)$ & $(\AA)$ & $(\mathrm{mag})$ \\
\hline May 13, 2007 & 54241 & $3.8 \pm 0.2$ & $-48 \pm 2.4$ & $11.52^{b}$ \\
Oct 15, 2007 & 54389 & $3.7 \pm 0.2$ & $-40 \pm 2.0$ & 11.53 \\
Oct 27, 2007 & 54401 & $3.5 \pm 0.2$ & $-28 \pm 1.7$ & 11.14 \\
Apr 01, 2008 & 54558 & $5.3 \pm 0.2$ & $-16 \pm 0.6$ & 10.09 \\
Apr 12, 2008 & 54569 & $6.1 \pm 0.2$ & $-20 \pm 0.6$ & 10.14 \\
Jun 10, 2008 & 54627 & $1.4 \pm 0.2$ & $-22 \pm 3.0$ & 11.96 \\
Jun 18, 2008 & 54635 & $1.2 \pm 0.2$ & $-19 \pm 3.0$ & 12.34 \\
Apr 24, 2010 & 55310 & $5.5 \pm 0.2$ & $-29 \pm 1.3$ & 10.63 \\
Jul 20, 2010 & 55397 & $5.1 \pm 0.2$ & $-25 \pm 1.6$ & 10.58 \\
Aug 08, 2010 & 55416 & $5.9 \pm 0.2$ & $-31 \pm 1.8$ & 10.63 \\
\hline
\end{tabular}

the former. Indeed, the J-band flux is composed of the direct radiation from the hot spot and an indirect brightening due to the radiation from the innermost regions of the circumstellar disk. Since PV Cep is embedded in an extremely massive disk (Hamidouche 2010), seen at large inclination, this effect has to be accounted for and likely represents the additional contribution that makes the continuum variation larger than the line emission. Similar anticorrelation results are given by Cohen et al. (1981), Magakian \& Movsessian (2001), and Acosta-Pulido et al. (2007).

This occurrence tends to rule out the variable extinction as cause of the observed variability that affects to the same extent both lines and continuum, since in that case a constant value of the EW should be expected for any continuum fluctuation. Even more a selective obscuration can be also ruled out. In fact, it should be located at the dust condensation zone, very close to the star and, as such, it should obscurate the stellar photosphere more than the accretion columns and the wind regions. On the contrary, accretion (or mass loss) processes are compatible with the observed behaviour, since they respond with different laws (Muzerolle et al. 1998), and maybe with different lags, to the continuum variations. These latter, once ruled out any extinction variation are unanimously attributed to hot spots on the stellar surface due to the 
shocks that originate at the base to the columns of the infalling matter. Being the line fluxes well correlated with the increasing continuum, they will depend on accretion processes: such a dependence may be direct if lines originate in the accretion columns, or indirect if lines originate in a stellar wind whose mass loss rate $\left(\dot{M}_{w i n d}\right)$ is related to the mass accretion rate $\left(\dot{M}_{a c c}\right)$ by invoking the rough proportion $\dot{M}_{\text {wind }} / \dot{M}_{a c c} \lesssim 0.1$ (Shu et al. 2000; Königl \& Pudritz 2000). Therefore we can assume that the total HI recombination line flux is the sum of different contributions: from accretion, from wind and, possibly, from other components (e.g. an HII circumstellar region). During strong accretion events, that increase the source continuum emission, only one contribution to the line emission dominates (correlation depicted in Figure 3), but when the impulsive accretion stops, namely when the continuum drops below a given threshold (in the present case $\mathrm{J}$ mag $\gtrsim 12$ ), line emission strength comes back to its original (lower) value, practically independent on the continuum value, and the EW value suddenly changes (see open symbols in Figure 3). In principle, alternative interpretations could exist: the proposed one is well compatible with the observational data and reconciles the two apparently unconsistent data points. However, to firmly confirm the presented scenario we still needs additional data resulting from the photometric and spectroscopic monitoring of relatively strong (more then 1-2 mag) variations of PV Cep or other targets. Indeed, one aspect that would deserve a deeper investigation is the following: by looking at Figures 4 and 1 , we can realize that the EW value reached immediately after the accretion event (that indicated by open symbols) is very similar to that corresponing to observations taken 100 days before, at an outburst peak. According to the proposed scenario, any EW value is associable to a continuum value lesser than a threshold, hence the observed EW resemblance is remarkable, but, plausibly, fortuitous.

\section{$5 \quad$ Final remarks}

The case presented here for a single young source (PV Cep) aims at fostering systematic photometric and spectroscopic monitoring intended as an observational method of investigation on how the mechanisms responsible for continuum and line emission are (inter-)related. Unfortunately, the proposed technique is not widely exploited since a frequent (weekly) monitoring (on years time-scale) is not easily doable both on large telescopes affected by a heavy oversubscription and on smaller telescopes often not equipped with the needed instrumentation. It would be very fruitful if small telescopes (1-2m class), but properly equipped, were dedicated to similar projects even for a small fraction $(<10 \%)$ of their observing time. 


\section{References}

- Acosta-Pulido, J.A. et al. 2007, AJ, 133, 2020

- Aspin, C. 2011, AJ, 141:196

- Cohen, M., Kuhi, L., Harlan, E. \& Spinard, H. 1981, ApJ 245, 920

- D'Alessio, F., et al. 2000 Proc. of the SPIE Symp. on Astronomical Telescopes and Instrumentation, eds. M. Iye \& A.F.M. Moorwood, 4008, 748

- Hamidouche, M. 2010 ApJ 722, 204

- Hartmann, L., \& Kenyon, S. 1985, ApJ, 299, 462

- Hillenbrand et al. 2012, arXiv 1208.2066

- Königl, A. \& Pudritz, R.E. 2000 Protostars and Planets IV - University of Arizona Press; eds V.Mannings, A.P.Boss, S.S Russell, p.759

- Kun, M. et al. 2011, MNRAS, 413, 2689

- Lorenzetti, D. et al. 2011, ApJ, 732:69

- Lorenzetti, D., Giannini, T., Larionov, V.M., Kopatskaya, E., Arkharov, A.A., De Luca, M., \& Di Paola, A. 2007, ApJ, 665, 1193

- Lorenzetti, D., Larionov, V.M., Giannini, T., Arkharov, A.A., Antoniucci, S., Nisini, B., \& Di Paola, A. 2009, ApJ, 693, 1056

- Magakian, T, \& Movsessian, T.A. 2001 Astrophysics 44, 419

- Muzerolle, J., Hartmann, L., \& Calvet, N. 1998 AJ, 116, 2965

- Reipurth, B., \& Aspin, C. 2004, ApJ 606, L119

- Reipurth, B., \& Aspin, C. 2010 Proc. of the Conference dedicated to Viktor Ambartsumian's 100th anniversary, Yerevan 15-18 Sep 2008 in Yerevan, eds H.A. Harutyunian, A.M. Mickaelian \& Y. Terzian, p. 19

- Shu, F.H., Najita, J.R., Ostriker, E., Wilkin, F., Ruden, S., \& Lizano, S. 1994, ApJ 429, 781

- Shu, F.H., Najita, J.R., Shang, -H. \& Li, Z.-Y. 2000 Protostars and Planets IV - University of Arizona Press; eds V.Mannings, A.P.Boss, S.S Russell, p.789

- Skrutskie, M. F., Meyer, M. R., Whalen, D., \& Hamilton, C. 1996, AJ 112, 2168 


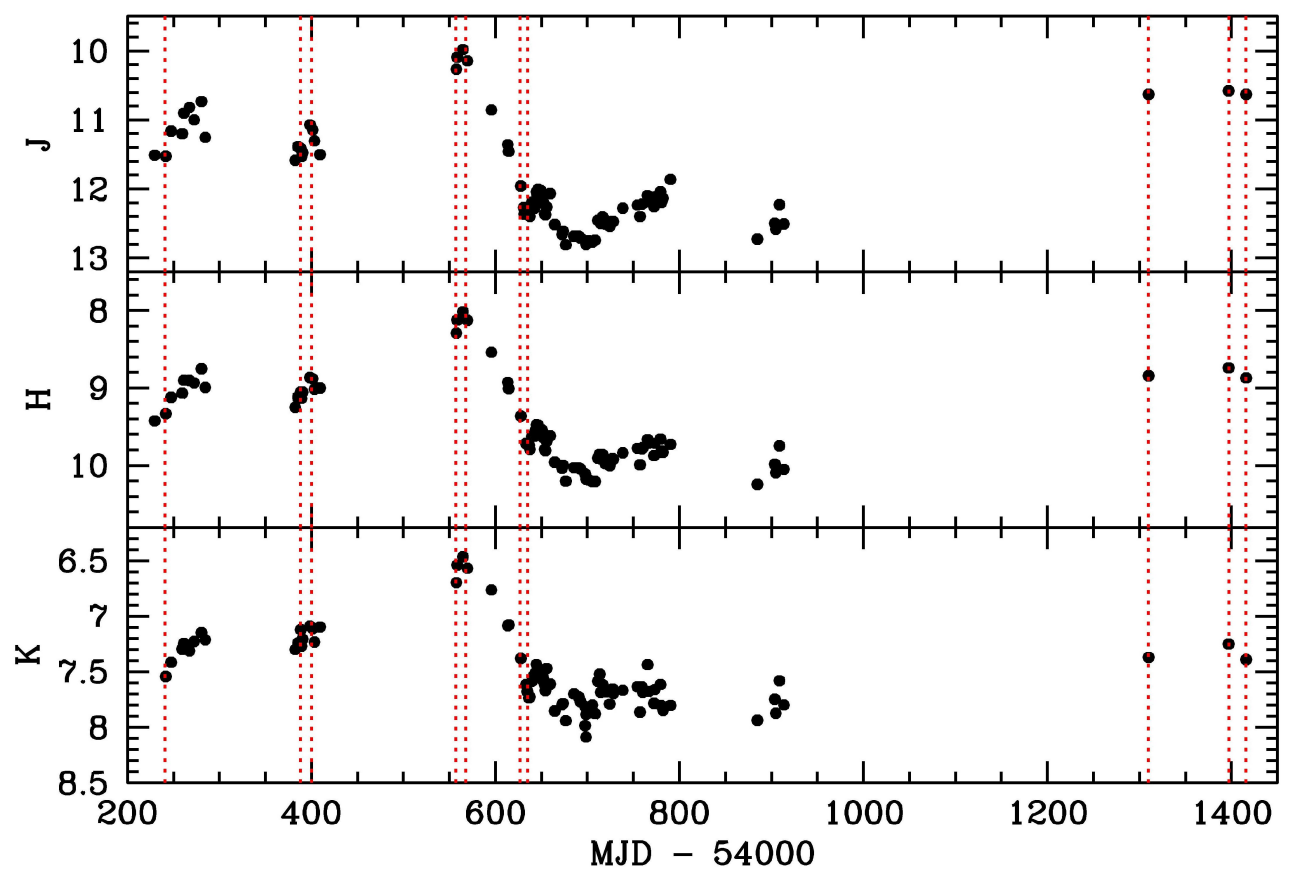

Fig. 1. PV Cep near-IR light curves vs. MJD (Modified Julian Date). The errors of data points are comparable to or lesser than $0.03 \mathrm{mag}$. Vertical red lines correspond to those dates when, beside JHK photometry, also a near-IR spectrum was taken. 


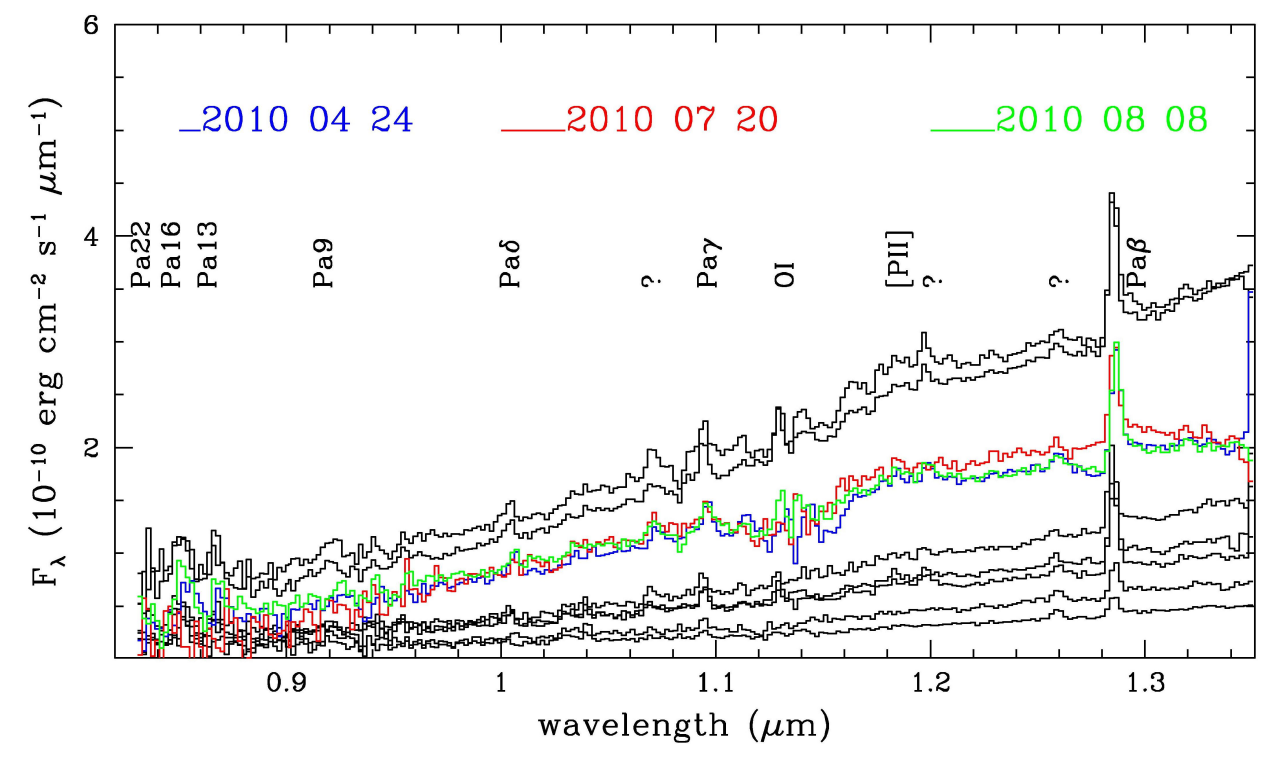

Fig. 2. The newly obtained near-IR spectra of PV Cep are depicted in colours (red, green, magenta). For comparison purposes, the already presented spectra are also plotted (in black). 


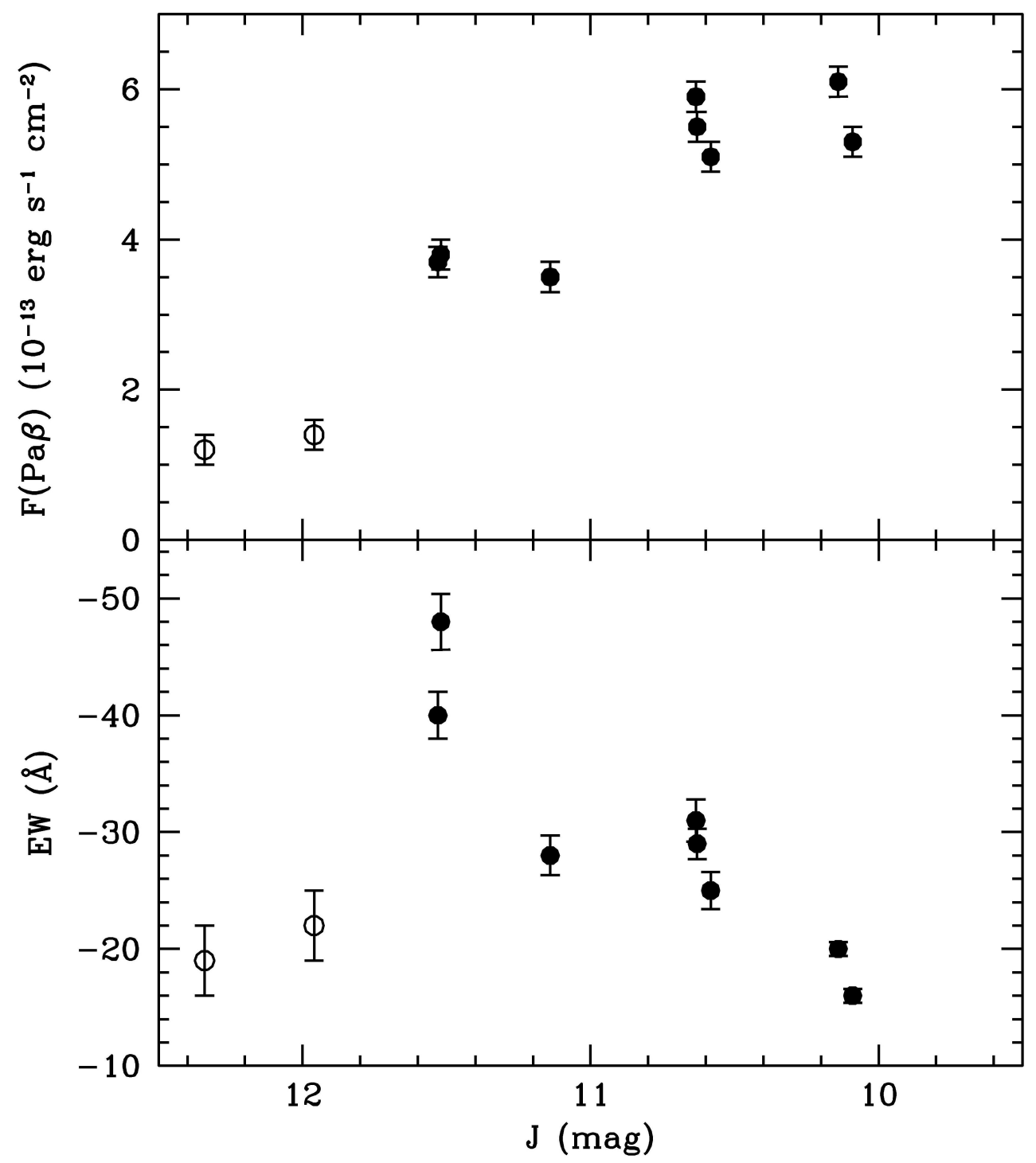

Fig. 3. Upper panel. Distribution of the $\mathrm{Pa} \beta$ fluxes extracted from the different spectra of PV Cep as a function of its J magnitude. Lower panel. Same as before, but in terms of Equivalent Widths; as usual, EW have negative values because line are in emission. Open symbols refer to the spectra taken just after a rapid fading of the source (see text and Figure 4). 


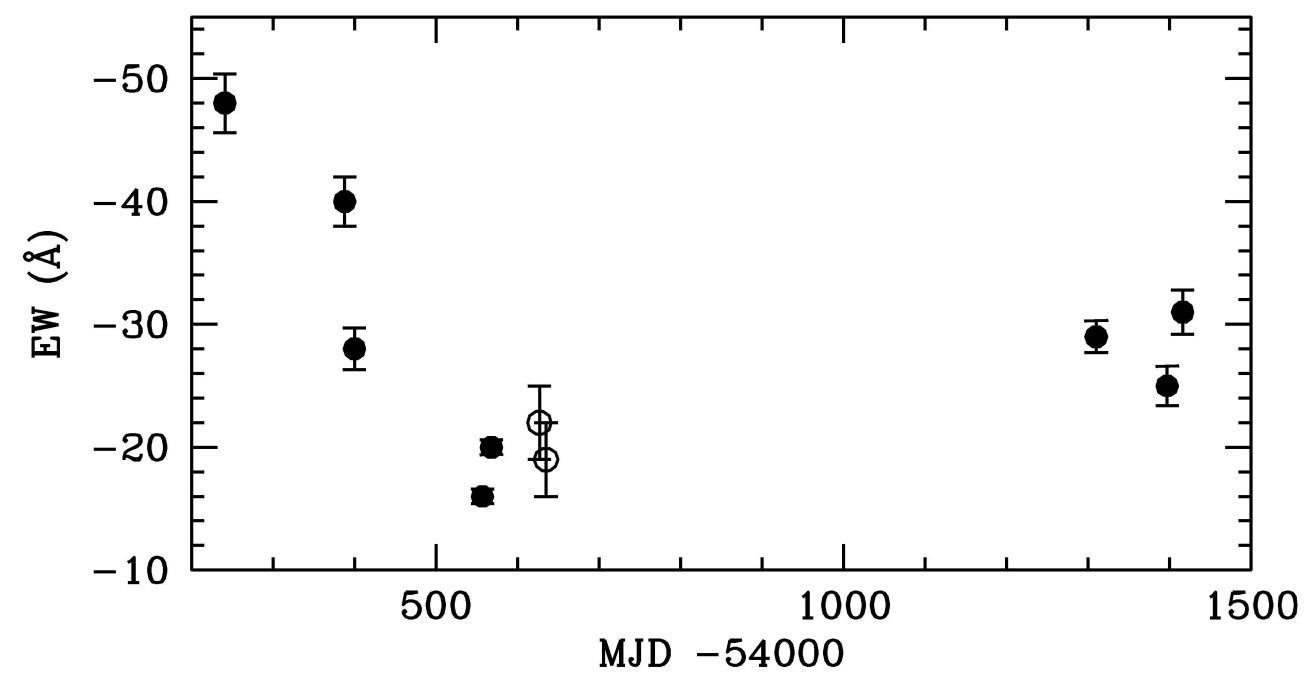

Fig. 4. EW derived from $\mathrm{Pa} \beta$ spectroscopy as a function of time. As in Figure 3 the same couple of data points is represented with open symbols. 